\title{
Positive Solutions for Coupled Nonlinear Fractional Differential Equations
}

\author{
Wenning Liu, ${ }^{1}$ Xingjie Yan, ${ }^{1}$ and Wei $\mathrm{Qi}^{2}$ \\ ${ }^{1}$ Department of Mathematics, China University of Mining and Technology, Xuzhou, Jiangsu 221008, China \\ ${ }^{2}$ Department of Physics, China University of Mining and Technology, Xuzhou, Jiangsu 221008, China \\ Correspondence should be addressed to Xingjie Yan; yanxingjie04@gmail.com
}

Received 25 January 2014; Accepted 1 May 2014; Published 12 May 2014

Academic Editor: Yongkun Li

Copyright (c) 2014 Wenning Liu et al. This is an open access article distributed under the Creative Commons Attribution License, which permits unrestricted use, distribution, and reproduction in any medium, provided the original work is properly cited.

\begin{abstract}
We consider the existence of positive solutions for a coupled system of nonlinear fractional differential equations with integral boundary values. Assume the nonlinear term is superlinear in one equation and sublinear in the other equation. By constructing two cones $K_{1}, K_{2}$ and computing the fixed point index in product cone $K_{1} \times K_{2}$, we obtain that the system has a pair of positive solutions. It is remarkable that it is established on the Cartesian product of two cones, in which the feature of two equations can be opposite.
\end{abstract}

\section{Introduction}

Fractional calculus is a very old concept dating back to 17th century; it involves fractional integration and fractional differentiation. At the first stage, fractional calculus theory is mainly focused on pure mathematical fields. In the last few decades, fractional differential equations and fractional integration equations have found many applications in various fields, such as science and engineering, physics, chemistry, biology, economics, and signal and image processing; for details, see [1-5]. In recent years, fractional differential equations have attracted increasing interests for their extensive applications, which leads to intensive development of the theory of fractional calculus. And more and more results about the existence and uniqueness of solutions appear. We can refer to [6] for the latest studies of fractional calculus.

The existence theory for initial value problems has been paid considerable attentions by many authors; see $[3,7$, $8]$ and the references cited therein. Also the existence of positive solutions for boundary value problem of nonlinear fractional differential equation has attracted attentions from many researches; see [9-14]. By using the cone extension method Feng et al. [15] studied the existence of solutions for higher-order nonlinear fractional differential equation with integral boundary conditions:

$$
\begin{gathered}
D_{0+}^{\alpha} u(t)+g(t) f(t, u(t))=0, \quad 0<t<1, \\
u(0)=u^{\prime}(0)=\cdots=u^{(n-2)}(0)=0, \\
u(1)=\int_{0}^{1} h(t) u(t) d t,
\end{gathered}
$$

where $D_{0+}^{\alpha}$ is the standard Riemann-Liouville fractional derivative of order $n-1<\alpha<n, n \geq 3, g \in \mathscr{C}((0,1),(0,+\infty))$ and $g$ may be singular at $t=0$ or $t=1, h \in L^{1}(0,1)$ is nonnegative, and $f \in \mathscr{C}((0,1) \times(0,+\infty),(0,+\infty))$.

In recent years, many authors have established the existence and uniqueness for solutions of some systems of nonlinear fractional differential equations; readers can see [16-21]. For example, by using the alternative of LeraySchauder theorem method Wang et al. [22] obtained the existence and uniqueness of positive solution of nonzero 
boundary values problem for a coupled system of nonlinear fractional differential equations:

$$
\begin{array}{cc}
D_{0+}^{\alpha} u(t)=f(t, v(t)), & 0<t<1, \\
D_{0+}^{\beta} v(t)=g(t, u(t)), & 0<t<1, \\
u(0)=0, & u(1)=a u(\xi), \\
v(0)=0, & v(1)=b v(\xi),
\end{array}
$$

where $1<\alpha, \beta<2,0 \leq a \leq b \leq 1,0<\xi<1$, $f, g:(0,1) \times \mathbb{R} \times \mathbb{R} \rightarrow \mathbb{R}$ are given continuous functions and $D_{0+}^{\alpha}$ is the standard Riemann-Liouville fractional derivative. By using the cone extension and cone compression Zhao et al. [23] studied the existence and nonexistence of positive solutions for a class of third order boundary value problem with integral boundary conditions in Banach spaces:

$$
\begin{gathered}
x^{\prime \prime \prime}+f(t, x(t))=0, \quad t \in J, \\
x(0)=0, \quad x^{\prime \prime}(0)=0, \quad x(1)=\int_{0}^{1} g(t) x(t) d t .
\end{gathered}
$$

Or

$$
\begin{gathered}
x^{\prime \prime \prime}+f(t, x(t))=0, \quad t \in J, \\
x(0)=\int_{0}^{1} g(t) x(t) d t, \quad x^{\prime \prime}(0)=0, \quad x(1)=0,
\end{gathered}
$$

where $J=(0,1), f \in \mathscr{C}((0,1) \times P, P), g \in L^{1}(0,1)$ is nonnegative and $D_{0+}^{\alpha}$ is the standard Riemann-Liouville fractional derivative; $P$ is a cone.

By using the cone extension and cone compression method Yang et al. [24] established sufficient conditions for the existence and nonexistence of positive solutions for a general class with integral boundary value problem for a coupled system of fractional equations:

$$
\begin{gathered}
D_{0+}^{\alpha} u(t)+a(t) f(t, v(t))=0, \quad 0<t<1, \\
D_{0+}^{\beta} v(t)+b(t) g(t, u(t))=0, \quad 0<t<1, \\
u(0)=0, \quad u(1)=\int_{0}^{1} \phi(t) u(t) d t, \\
v(0)=0, \quad v(1)=\int_{0}^{1} \varphi(t) v(t) d t,
\end{gathered}
$$

where $1<\alpha, \beta \leq 2, a, b \in \mathscr{C}((0,1),(0,+\infty)), f, g \in \mathscr{C}((0,1) \times$ $(0,+\infty),(0,+\infty))$, and $\phi, \varphi \in L^{1}(0,1)$ are nonnegative and $D_{0+}^{\alpha}$ is the standard Riemann-Liouville fractional derivative.

However, all these works are obtained by getting a fixed point of a completely continuous operator in a cone of product Banach spaces $X_{1} \times X_{2}$. Thus, there is no essential difference between we consider the positive solution of coupled equations and positive solution of single equation. Furthermore, the authors cannot guarantee the obtained pair of positive solutions are all positive; maybe one is positive and another is zero. The features of nonlinear term are the same, which means the growth properties of nonlinear term are similar.

Motivated by the above mentioned works, in this paper, we consider the existence of positive solution to boundary value problem for a coupled system of nonlinear fractional differential equations as follows:

$$
\begin{gathered}
D_{0+}^{\alpha} u(t)+f(t, u(t), v(t))=0, \quad 0<t<1, \\
D_{0+}^{\beta} v(t)+g(t, u(t), v(t))=0, \quad 0<t<1, \\
u(0)=0, \quad u(1)=\int_{0}^{1} \phi(t) u(t) d t, \\
v(0)=0, \quad v(1)=\int_{0}^{1} \varphi(t) v(t) d t,
\end{gathered}
$$

where $1<\alpha, \beta \leq 2, f, g \in \mathscr{C}((0,1) \times(0,+\infty) \times$ $(0,+\infty),(0,+\infty))$, and $\phi, \varphi \in L^{1}(0,1)$ are nonnegative and $D_{0+}^{\alpha}$ is the standard Riemann-Liouville fractional derivative. $f$ and $g$ have different features. It is very difficult to deal with our problem if we directly use the usual method because the features of nonlinear term are different. In order to overcome the difficulties, by using the ideals in [25-27], we need to consider our problem on the Cartesian product of two cones in the space $\mathscr{C}(0,1)$; thus we can better exploit the feature of two equations. We choose a cone $K_{1} \times K_{2}$ in the Cartesian product of two cones in $\mathscr{C}(0,1) \times \mathscr{C}(0,1)$. By computing the fixed point index in $K_{1} \times K_{2}$, we obtain the existence of positive solution $(u, v)$ of problem (6) such that $u>0, v>0$.

\section{Preliminary}

In this section, we review some basic facts which are used throughout this paper. We can see $[10,16,25-29]$ for more details.

Definition 1. For a function $f:[a,+\infty) \rightarrow \mathbb{R}$, the RiemannLiouville fractional integral of order $\alpha>0$ is defined by

$$
I_{a+}^{\alpha} f(t)=\frac{1}{\Gamma(\alpha)} \int_{a}^{t}(t-s)^{\alpha-1} f(s) d s,
$$

and the right-hand side is pointwise defined on $[a,+\infty)$, where $a \in \mathbb{R}$ and $\Gamma$ is the gamma function.

Definition 2. For a function $f:[a,+\infty) \rightarrow \mathbb{R}$, the RiemannLiouville fractional derivative of order $\alpha>0$ is given by

$$
D_{a+}^{\alpha} f(t)=\frac{1}{\Gamma(\alpha)} \frac{d^{n}}{d t^{n-1}} \int_{a}^{t}(t-s)^{n-\alpha-1} f(s) d s,
$$

and the right-hand side is pointwise defined on $[a,+\infty)$, where $a \in \mathbb{R}, n=[\alpha]+1$ and $\Gamma$ is the gamma function.

Let $E$ be a real Banach space with norm \|\| and let $A$ : $E \rightarrow E$ be an operator. A nonempty, closed, convex set $P \subset E$ is said to be a cone provided the following: (i) if $x \in P$ and $\lambda \geq 0$, then $\lambda x \in P$; (ii) if $x \in P$ and $-x \in P$, then $x=0$ 
are satisfied. $u$ is said to be a fixed point of $A$, if $u=A u$. $\Omega$ is a bounded open subset of $E$ with boundary $\partial \Omega$. Denote $i(A, P \cap \Omega, P)$ the fixed point index of $A$.

Lemma 3 (see [25-27]). Let $E$ be a Banach space and let $K_{i} \subset$ $E(i=1,2)$ be a closed convex cone in $E$. For $r_{i}>0(i=1,2)$, denote $K_{r_{i}}=\left\{u \in K_{i} \mid\|u\|<r_{i}\right\}, \partial K_{i}=\left\{u \in K_{i} \mid\|u\|=r_{i}\right\}$. Suppose $A_{i}: K_{i} \rightarrow K_{i}$ is completely continuous. If $u_{i} \neq A_{i} u_{i}$, $\forall u_{i} \in \partial K_{i}$, then

$$
i\left(A, K_{r_{1}} \times K_{r_{2}}, K_{1} \times K_{2}\right)=i\left(A_{1}, K_{r_{1}}, K_{1}\right) \cdot i\left(A_{2}, K_{r_{2}}, K_{2}\right),
$$

where $A(u, v)=\left(A_{1} u, A_{2} v\right)$ and for all $(u, v) \in\left(K_{1} \times K_{2}\right)$.

In order to prove the existence of solution for problem (6), we can translate it into obtaining a fixed point of operator. Then we use the topological method to deal with it.

Theorem 4. Let $E$ be a Banach space and let $K_{1}, K_{2} \subset E$ be a closed convex cone in E. Denote $K_{r_{i}}=\left\{u \in K_{i} \mid\|u\|<r_{i}\right\}$, $\partial K_{i}=\left\{u \in K_{i}\|u\|=r_{i}\right\}$, where $r>0, i=1,2$. Let $D=$ $\left(K_{R_{1}} \backslash \overline{K_{r_{1}}}\right) \times\left(K_{R_{2}} \backslash \overline{K_{r_{2}}}\right)$. Suppose $T: K_{1} \times K_{2} \rightarrow K_{1} \times K_{2}$ is completely continuous and $T(u, v)=\left(T_{1}(u, v), T_{2}(u, v)\right)$. For $\lambda \in[0,1]$, let

$$
\begin{aligned}
& H_{\lambda, 1}(u, v)=\lambda T_{1}(u, v)+(1-\lambda) T_{1}(u, 0), \\
& H_{\lambda, 2}(u, v)=\lambda T_{2}(u, v)+(1-\lambda) T_{2}(0, v) .
\end{aligned}
$$

If $H_{\lambda, 1}, H_{\lambda, 2}$ satisfy the following conditions:

(i) $H_{\lambda, 1}$ is uniformly cone extension about $v \in K_{R_{2}}$ at $K_{R_{1}}$ । $\overline{K_{r_{1}}}$, that is to say,

$$
\begin{gathered}
\left\|H_{\lambda, 1}\right\|<\|u\|, \quad \forall u \in \partial K_{r_{1}}, \\
\left\|H_{\lambda, 1}\right\|>\|u\|, \quad \forall u \in \partial K_{R_{1}} ;
\end{gathered}
$$

(ii) $H_{\lambda, 2}$ is uniformly cone compression about $u \in K_{R_{1}}$ at $K_{R_{2}} \backslash \overline{K_{r_{2}}}$, that is to say,

$$
\begin{gathered}
\left\|H_{\lambda, 2}\right\|>\|v\|, \quad \forall v \in \partial K_{r_{2}}, \\
\left\|H_{\lambda, 2}\right\|<\|v\|, \quad \forall v \in \partial K_{R_{2}},
\end{gathered}
$$

then $i\left(T, D, K_{1} \times K_{2}\right)=-1$. Thus the mapping $T$ in the region $D$ has a fixed point.

Proof. Following a similar procedure to the method given in [25-27], we present a proof. We suppose that $T_{1}(u, 0)=A_{1} u$ and $T_{2}(0, v)=A_{2} v$. Let $A(u, v)=\left(A_{1} u, A_{2} v\right)$. For $\lambda \in[0,1]$, for any $(u, v) \in K_{1} \times K_{2}$, set

$$
H((u, v), \lambda)=\lambda T(u, v)+(1-\lambda) A(u, v) .
$$

Since $T$ is completely continuous, hence the mapping $H$ is also completely continuous. By Lemma 3 and homotopy invariance of fixed point index (see $[7,28])$, we have

$$
\begin{aligned}
& i\left(T, D, K_{1} \times K_{2}\right) \\
& \quad=i\left(A_{1}, K_{R_{1}} \backslash \overline{K_{r_{1}}}, K_{1}\right) \cdot i\left(A_{2}, K_{R_{2}} \backslash \overline{K_{r_{2}}}, K_{2}\right) .
\end{aligned}
$$

Next, we compute $i\left(T, D, K_{1} \times K_{2}\right)$. By assumptions (i) and (ii), also using the properties of fixed point index (see $[7,28]$ ), we have

$$
\begin{aligned}
& i\left(A_{1}, K_{r_{1}}, K_{1}\right)=i\left(A_{2}, K_{R_{2}}, K_{2}\right)=1, \\
& i\left(A_{1}, K_{R_{1}}, K_{1}\right)=i\left(A_{2}, K_{r_{2}}, K_{2}\right)=0 .
\end{aligned}
$$

Thanks to (15), we obtain

$$
\begin{aligned}
i\left(T, D, K_{1} \times K_{2}\right) \\
=i\left(A_{1}, K_{R_{1}} \backslash \overline{K_{r_{1}}}, K_{1}\right) \cdot i\left(A_{2}, K_{R_{2}} \backslash \overline{K_{r_{2}}}, K_{2}\right) \\
=\left(i\left(A_{1}, K_{R_{1}}, K_{1}\right)-i\left(A_{1}, \overline{K_{r_{1}}}, K_{1}\right)\right) \\
\quad\left(i\left(A_{2}, K_{R_{2}}, K_{2}\right)-i\left(A_{2}, \overline{K_{r_{2}}}, K_{2}\right)\right) \\
=(0-1) \cdot(1-0)=-1 .
\end{aligned}
$$

The proof is complete.

Remark 5. If $H_{\lambda, 1}, H_{\lambda, 2}$ were all cone extension or cone compression, we can obtain the same conclusions.

The following lemmas are necessary to prove the existence of positive solution for problem (6).

Lemma 6 (see [15]). Assume that $\int_{0}^{1} \phi(t) u(t) d t \neq 1$. Then for any $\psi \in \mathscr{C}(0,1)$, the solution of boundary value problem

$$
\begin{gathered}
D_{0+}^{\alpha} u(t)+\psi(t)=0, \quad 0<t<1, \\
u(0)=0, \quad u(1)=\int_{0}^{1} \phi(t) u(t) d t
\end{gathered}
$$

is given by $u(t)=\int_{0}^{1} G_{1 \alpha}(t, s) \psi(s) d s$, where $G_{1 \alpha}(t, s)=$ $G_{2 \alpha}(t, s)+G_{3 \alpha}(t, s)$,

$$
\begin{aligned}
& G_{2 \alpha}(t, s)= \begin{cases}\frac{t^{\alpha-1}(1-s)^{\alpha-1}-(t-s)^{\alpha-1}}{\Gamma(\alpha)}, & 0 \leq s \leq t \leq 1 . \\
\frac{t^{\alpha-1}(1-s)^{\alpha-1}}{\Gamma(\alpha)}, & 0 \leq t \leq s \leq 1 .\end{cases} \\
& G_{3 \alpha}(t, s)=\frac{t^{\alpha-1}}{1-\int_{0}^{1} \phi(t) t^{\alpha-1} d t} \int_{0}^{1} \phi(t) G_{2 \alpha}(t, s) d t .
\end{aligned}
$$

Lemma 7 (see [15]). If $\int_{0}^{1} \phi(t) t^{\alpha-1} d t \in[0,1)$, then Green's function $G_{1 \alpha}(t, s)$ has the following properties:

(i) $G_{1 \alpha}(t, s) \geq 0$ is continuous for any $t, s \in[0,1]$, $G_{1 \alpha}(t, s)>0$ for any $t, s \in(0,1)$;

(ii) $G_{1 \alpha}(t, s) \leq G_{1 \alpha}(s)$ for any $t, s \in[0,1]$ and $\min _{t \in[\theta, 1-\theta]} G_{1 \alpha}(t, s) \geq \gamma_{\theta} G_{1 \alpha}(s)$, where $G_{1 \alpha}(s)=$ $G_{2 \alpha}(s, s)+G_{3 \alpha}(1, s), \gamma_{\theta}=\theta^{\alpha-1}, \theta \in(0,1 / 2)$. 


\section{Main Results}

In this section, we will discuss the existence of positive solutions for problem (6). For a coupled system, many researchers transformed the problem into computing the fixed point index of the composition operator on the single cone in the Cartesian product space and the nonlinear terms in two equations have similar features. However it is very difficult to deal with our problem directly. Denote $\mathscr{C}^{+}[0,1]$ the continuous function in $[0,1]$ with nonnegative value. In order to make full use of the different nature of the two equations, we construct single cone $K_{i}$ and open set $K_{R_{i}} \backslash \overline{K_{r_{i}}}, i=1,2$ in $\mathscr{C}^{+}[0,1]$. Thus we transform the problem into computing the fixed point index of operator that is defined in Cartesian product of two cones in open set $D=\left(K_{R_{1}} \backslash \overline{K_{r_{1}}}\right) \times\left(K_{R_{2}} \backslash \overline{K_{r_{2}}}\right)$ of product cone $K_{1} \times K_{2}$.

3.1. The Transformation of the Problem. Firstly, we construct the relevant cone $K$ in Banach space $\mathscr{C}^{+}[0,1]$. Then we transform the problem into computing the fixed point index of Cartesian product of cone with the nature of analytical property of Green's function.

Following, we introduce notations:

$$
\mathscr{C}^{+}[0,1]=\{u \in \mathscr{C}[0,1] \mid u(t) \geq 0, \forall t \in[0,1]\},
$$

with the norm $\|u\|=\max _{t \in[0,1]}\{|u(t)|\}$. Clearly, it is a Banach space. Define

$$
\begin{array}{r}
K_{1}=\left\{u \in \mathscr{C}^{+}[0,1] \mid u(t) \geq\left(\frac{1}{2}\right)^{\alpha-1}\|u(t)\|,\right. \\
\left.\forall t \in[\theta, 1-\theta], \theta \in\left(0, \frac{1}{2}\right)\right\}, \\
K_{2}=\left\{u \in \mathscr{C}^{+}[0,1] \mid u(t) \geq\left(\frac{1}{2}\right)^{\beta-1}\|u(t)\|,\right. \\
\left.\forall t \in[\theta, 1-\theta], \theta \in\left(0, \frac{1}{2}\right)\right\} .
\end{array}
$$

For $\lambda \in[0,1], u, v \in \mathscr{C}^{+}[0,1]$, we define the operators

$$
\begin{gathered}
T_{\lambda, 1}(u, v): \mathscr{C}^{+}[0,1] \times \mathscr{C}^{+}[0,1] \longrightarrow \mathscr{C}^{+}[0,1], \\
T_{\lambda, 2}(u, v): \mathscr{C}^{+}[0,1] \times \mathscr{C}^{+}[0,1] \longrightarrow \mathscr{C}^{+}[0,1], \\
T_{\lambda}(u, v): \mathscr{C}^{+}[0,1] \times \mathscr{C}^{+}[0,1] \longrightarrow \mathscr{C}^{+}[0,1] \times \mathscr{C}^{+}[0,1],
\end{gathered}
$$

as follows:

$$
\begin{aligned}
& T_{\lambda, 1}(u, v)=\int_{0}^{1} G_{1 \alpha}(t, s)( \lambda f(s, u(s), v(s)) \\
&+(1-\lambda) f(s, u(s), 0)) d s, \\
& T_{\lambda, 2}(u, v)=\int_{0}^{1} G_{1 \beta}(t, s)( \lambda g(s, u(s), v(s)) \\
&+(1-\lambda) g(s, 0, v(s))) d s, \\
& T_{\lambda}(u, v)(t)=\left(T_{\lambda, 1}(u, v)(t), T_{\lambda, 2}(u, v)(t)\right) .
\end{aligned}
$$

Then we translate that problem (6) possesses a pair of solutions $(u, v)$ if and only if $T_{\lambda}(u, v)(t)$ have a fixed point in $K_{1} \times K_{2}$.

Lemma 8. The mapping $T_{\lambda}: K_{1} \times K_{2} \rightarrow K_{1} \times K_{2}$ as mentioned above is completely continuous.

Proof. For any $(u, v) \in K_{1} \times K_{2}$, we want to prove $T_{\lambda}(u, v) \in$ $K_{1} \times K_{2}$, that is, to prove $T_{\lambda, 1}(u, v) \in K_{1}$ and $T_{\lambda, 2}(u, v) \in K_{2}$. By the preceding definition and the nature of Green's function, we have for $t \in[\theta, 1-\theta], \theta \in(0,1 / 2)$

$$
\begin{aligned}
& T_{\lambda, 1}(u, v) \\
& =\int_{0}^{1} G_{1 \alpha}(t, s)(\lambda f(s, u(s), v(s)) \\
& +(1-\lambda) f(s, u(s), 0)) d s \\
& \geq \int_{0}^{1} \gamma_{\theta} G_{1 \alpha}(s)(\lambda f(s, u(s), v(s)) \\
& \quad+(1-\lambda) f(s, u(s), 0)) d s \\
& =\gamma_{\theta} \int_{0}^{1} G_{1 \alpha}(s)(\lambda f(s, u(s), v(s)) \\
& \geq\left(\frac{1}{2}\right)^{\alpha-1} \int_{0}^{1} G_{1 \alpha}(t, s)(\lambda f(s, u(s), v(s)) \\
& \quad+(1-\lambda) f(s, u(s), 0)) d s \\
& \geq\left(\frac{1}{2}\right)^{\alpha-1}\left\|T_{\lambda, 1}(u, v)\right\| .
\end{aligned}
$$

Similarly, $T_{\lambda, 2}(u, v) \geq(1 / 2)^{\beta-1}\left\|T_{\lambda, 2}(u, v)\right\|, t \in[\theta, 1-\theta]$, $\theta \in(0,1 / 2)$. Thus, $T_{\lambda, 1}(u, v) \in K_{1}, T_{\lambda, 2}(u, v) \in K_{2}$, and $T_{\lambda}\left(K_{1} \times K_{2}\right) \subset K_{1} \times K_{2}$.

It is easy to prove that $T_{\lambda}(u, v)$ is continuous in $K_{1} \times K_{2}$.

So as Arzela-Ascoli theorem, we prove that $T_{\lambda}: K_{1} \times$ $K_{2} \rightarrow K_{1} \times K_{2}$ is completely continuous.

3.2. The Main Result and Its Proof. Now, we present our main result.

Theorem 9. Assume that the nonlinear terms of the problem (6) $f$ and $g$ meet the following conditions:

$$
\left(H_{1}\right)\left\{\begin{array}{c}
\left(a_{1}\right) \lim _{u \rightarrow 0^{+}} \sup \max _{t \in[0,1]} \frac{f(t, u, v)}{u}=0 \\
\text { is established consistent about } v \in \mathbb{R}^{+}, \\
\left(b_{1}\right) \lim _{u \rightarrow+\infty} \inf \min _{t \in[0,1]} \frac{f(t, u, v)}{u}=+\infty \\
\text { is established consistent about } v \in \mathbb{R}^{+},
\end{array}\right.
$$




$$
\begin{aligned}
& \left(H_{2}\right)\left\{\begin{array}{r}
\left(a_{2}\right) \lim _{v \rightarrow 0^{+}} \inf \min _{t \in[0,1]} \frac{g(t, u, v)}{v}=+\infty \\
\text { is established consistent about } u \in \mathbb{R}^{+}, \\
\left(b_{2}\right) \lim _{v \rightarrow+\infty} \sup \max _{t \in[0,1]} \frac{g(t, u, v)}{v}=0 \\
\text { is established consistent about } u \in \mathbb{R}^{+},
\end{array}\right. \\
& \left(H_{3}\right) \lim _{u \rightarrow+\infty} \sup \max _{t \in[0,1]} g(t, u, v)=h(v)
\end{aligned}
$$$$
\text { is established consistent about } v \in[0, M] \text {, }
$$

where $h(v)$ is continuous in $\mathbb{R}^{+}$and $M$ is a constant.

Then the problem (6) has at least a pair of positive solutions.

Proof. We select an appropriate open set $D=\left(K_{R_{1}} \backslash \overline{K_{r_{1}}}\right) \times$ $\left(K_{R_{2}} \backslash \overline{K_{r_{2}}}\right)$ in the product of cone $K_{1} \times K_{2}$; then we will verify that the solution operator meets the conditions of Theorem 4 .

In the following, we separate our proof into four steps.

Step 1. Select $r_{1}>0$, in such a fashion that $\left\|T_{\lambda, 1}(u, v)\right\|<\|u\|$, where $\lambda \in[0,1],(u, v) \in \partial K_{r_{1}} \times K_{1}$. Based on assuming $\left(H_{1}\right)\left(a_{1}\right)$, for $\varepsilon_{1}=\left(\int_{0}^{1} G_{1 \alpha}(s) d s\right)^{-1}>0$, there exists $r_{1}>0$, such that $f(t, u, v)<\varepsilon_{1} u$, for all $t \in[0,1], 0 \leq u \leq r_{1}, v \geq 0$. Then when $\lambda \in[0,1],(u, v) \in \partial K_{r_{1}} \times K_{1}$, we have for $t \in[0,1]$

$$
\begin{aligned}
& \left\|T_{\lambda, 1}(u, v)\right\| \\
& =\max _{t \in[0,1]} \mid \int_{0}^{1} G_{1 \alpha}(t, s)(\lambda f(s, u(s), v(s)) \\
& +(1-\lambda) f(s, u(s), 0)) d s \mid \\
& <\max _{t \in[0,1]} \int_{0}^{1} G_{1 \alpha}(s)\left|\lambda \varepsilon_{1} u(s)+(1-\lambda) \varepsilon_{1} u(s)\right| d s \\
& \leq \max _{t \in[0,1]} \int_{0}^{1} G_{1 \alpha}(s) \varepsilon_{1}|u(s)| d s \\
& \leq \varepsilon_{1} \int_{0}^{1} G_{1 \alpha}(s) \max _{t \in[0,1]}|u(s)| d s \\
& \leq \varepsilon_{1} \int_{0}^{1} G_{1 \alpha}(s) d s\|u\|=\|u\| .
\end{aligned}
$$

That is to say, $\left\|T_{\lambda, 1}(u, v)\right\|<\|u\|$, where $\lambda \in[0,1],(u, v) \in$ $\partial K_{r_{1}} \times K_{1}$.

Step 2. Select $R_{1}>r_{1}$; we claim that $\left\|T_{\lambda, 1}(u, v)\right\|>\|u\|$, where $\lambda \in[0,1],(u, v) \in \partial K_{R_{1}} \times K_{1}$. In view of the assumption $\left(H_{1}\right)\left(b_{1}\right)$, for $N_{1}=\left(\gamma_{\theta} \int_{0}^{1} G_{1 \alpha}(s) d s\right)^{-1}>0$, there exists $R_{0}>0$, such that $f(t, u, v)<N_{1} u$, for all $t \in[0,1], u \geq R_{0}, v \geq 0$. Let
$R_{1}>\max \left\{r_{1}, \gamma_{\theta} R_{0}\right\}$. Then when $\lambda \in[0,1],(u, v) \in \partial K_{R_{1}} \times K_{1}$, we have $t \in[0,1]$

$$
\begin{aligned}
& \left\|T_{\lambda, 1}(u, v)\right\| \\
& =\max _{t \in[0,1]} \mid \int_{0}^{1} G_{1 \alpha}(t, s)(\lambda f(s, u(s), v(s)) \\
& +(1-\lambda) f(s, u(s), 0)) d s \\
& >\max _{t \in[0,1]} \int_{0}^{1} \gamma_{\theta} G_{1 \alpha}(s)\left|\lambda N_{1} u(s)+(1-\lambda) N_{1} u(s)\right| d s \\
& \geq \max _{t \in[0,1]} \gamma_{\theta} \int_{0}^{1} G_{1 \alpha}(s) N_{1}|u(s)| d s \\
& \geq N_{1} \gamma_{\theta} \int_{0}^{1} G_{1 \alpha}(s) \max _{t \in(0,1)}|u(s)| d s \\
& \geq N_{1} \gamma_{\theta} \int_{0}^{1} G_{1 \alpha}(s) d s\|u\|=\|u\|
\end{aligned}
$$

That is to say, $\left\|T_{\lambda, 1}(u, v)\right\|>\|u\|$, where $\lambda \in[0,1],(u, v) \in$ $\partial K_{R_{1}} \times K_{1}$.

Step 3. Select $r_{2}>0$, such that $\left\|T_{\lambda, 2}(u, v)\right\|>\|v\|$, where $\lambda \epsilon$ $[0,1],(u, v) \in \partial K_{r_{2}} \times K_{2}$. In view of the assumption $\left(H_{2}\right)\left(a_{2}\right)$, for $N_{2}=\left(\gamma_{\theta} \int_{0}^{1} G_{1 \alpha}(s) d s\right)^{-1}>0$, there exists $r_{2}>0$, such that $g(t, u, v)>N_{2} v$, for all $t \in[0,1], 0 \leq v \leq r_{2}, u \geq 0$. Hence when $\lambda \in[0,1],(u, v) \in \partial K_{r_{2}} \times K_{2}$, we have for $t \in[0,1]$

$$
\begin{aligned}
& \left\|T_{\lambda, 2}(u, v)\right\| \\
& =\max _{t \in[0,1]} \mid \int_{0}^{1} G_{1 \beta}(t, s)(\lambda g(s, u(s), v(s)) \\
& +(1-\lambda) g(s, 0, v(s))) d s \\
& >\max _{t \in[0,1]} \int_{0}^{1} \gamma_{\theta} G_{1 \beta}(s)\left|\lambda N_{2} v(s)+(1-\lambda) N_{2} v(s)\right| d s \\
& \geq \max _{t \in[0,1]} \gamma_{\theta} \int_{0}^{1} G_{1 \beta}(s) N_{2}|v(s)| d s \\
& \geq N_{2} \gamma_{\theta} \int_{0}^{1} G_{1 \beta}(s) \max _{t \in(0,1)}|v(s)| d s \\
& \geq N_{2} \gamma_{\theta} \int_{0}^{1} G_{1 \alpha}(s) d s\|v\|=\|v\| .
\end{aligned}
$$

That is to say, $\left\|T_{\lambda, 2}(u, v)\right\|>\|v\|$, where $\lambda \in[0,1],(u, v) \in$ $\partial K_{r_{2}} \times K_{2}$.

Step 4. Select $R_{2}>r_{2}$, such that $\left\|T_{\lambda, 2}(u, v)\right\|<\|v\|$, where $\lambda \in[0,1],(u, v) \in \partial K_{R_{2}} \times K_{2}$. Based on the condition $\left(H_{2}\right)\left(b_{2}\right)$, for $\varepsilon_{2}=\left(\int_{0}^{1} G_{1 \alpha}(s) d s\right)^{-1}>0$, there exists $R^{\prime}>0$, such that $g(t, u, v)<\varepsilon_{2} v$, for all $t \in[0,1], v \geq R^{\prime}, u \geq 0$. Furthermore, 
paying attention to the assumptions $\left(H_{3}\right)$, we can select $R_{2}>$ $0, R_{2}$ which is sufficiently large and

$$
\max _{t \in[0,1]}\left\{g(t, u, v) \mid 0 \leq v \leq R^{\prime}, u \geq 0\right\}<\varepsilon_{2} R_{2}
$$

Thus $g(t, u, v)<\varepsilon_{2} R_{2}$, where $t \in[0,1], 0 \leq v \leq R_{2}, u \geq 0$. Hence when $\lambda \in[0,1],(u, v) \in \partial K_{R_{2}} \times K_{2}$, we have for $t \in$ $[0,1]$

$$
\begin{aligned}
& \left\|T_{\lambda, 2}(u, v)\right\| \\
& =\max _{t \in[0,1]} \mid \int_{0}^{1} G_{1 \beta}(t, s)(\lambda g(s, u(s), v(s)) \\
& +(1-\lambda) g(s, 0, v(s))) d s \\
& <\max _{t \in[0,1]} \int_{0}^{1} G_{1 \beta}(s)\left(\lambda \varepsilon_{2} R_{2}+(1-\lambda) \varepsilon_{2} R_{2}\right) d s \\
& \leq \max _{t \in[0,1]} \int_{0}^{1} G_{1 \beta}(s) \varepsilon_{2} R_{2} d s \\
& \leq \varepsilon_{2} R_{2} \int_{0}^{1} G_{1 \beta}(s) \max _{t \in[0,1]} v(s) d s \\
& \leq \varepsilon_{2} R_{2} \int_{0}^{1} G_{1 \alpha}(s) d s=\|v\| \text {. }
\end{aligned}
$$

That is to say, $\left\|T_{\lambda, 2}(u, v)\right\|<\|v\|$, where $\lambda \in[0,1],(u, v) \in$ $\partial K_{R_{2}} \times K_{2}$.

Combining with (25)-(29), we know the conditions of Theorem 4 are all established. Thus the completely continuous operator $T$ has a fixed point in $D$; that is to say, at least a pair of positive solutions exist for the problem (6). The proof is complete.

Remark 10. Binding (27), (29) and the assumption $\left(H_{1}\right)\left(a_{1}\right)$, we can get a nonnegative solution $(0, v)$ of the original equations (6). In fact, by (27) and (29), we know the fixed point index $i\left(T_{0,2}(u, v), K_{R_{2}} \backslash \overline{K_{r_{2}}}, K_{2}\right)=-1$; that is to say, the second equation of the original equations has a solution $(0, v)$. Further, by hypothesis $\left(H_{1}\right)\left(a_{1}\right)$, we have $f(t, 0, v(t))=0$, so the solution of the second equation is also the solution of the first equation.

\section{Conflict of Interests}

The authors declare that there is no conflict of interests regarding the publication of this paper.

\section{Acknowledgments}

This work was partly supported by the Fundamental Research Funds for the Central Universities Grant (2012LWN56) and the Nature Science Foundation of Jiangsu Province Grant (BK20130170).

\section{References}

[1] O. P. Agrawal, "Formulation of Euler-Lagrange equations for fractional variational problems," Journal of Mathematical Analysis and Applications, vol. 272, no. 1, pp. 368-379, 2002.

[2] Z. Bai and H. Lü, "Positive solutions for boundary value problem of nonlinear fractional differential equation," Journal of Mathematical Analysis and Applications, vol. 311, no. 2, pp. 495-505, 2005.

[3] D. Delbosco and L. Rodino, "Existence and uniqueness for a nonlinear fractional differential equation," Journal of Mathematical Analysis and Applications, vol. 204, no. 2, pp. 609-625, 1996.

[4] S. G. Samko, A. A. Kilbas, and O. I. Marichev, Fractional Integrals and Derivatives, Theorey and Applications, Gordon and Breach Science, Yverdon, Switzerland, 1993.

[5] S. Zhang, "Existence of positive solution for some class of nonlinear fractional differential equations," Journal of Mathematical Analysis and Applications, vol. 278, no. 1, pp. 136-148, 2003.

[6] J. T. Machado, V. Kiryakova, and F. Mainardi, "Recent history of fractional calculus," Communications in Nonlinear Science and Numerical Simulation, vol. 16, no. 3, pp. 1140-1153, 2011.

[7] H. Jafari and V. Daftardar-Gejji, "Positive solutions of nonlinear fractional boundary value problems using Adomian decomposition method," Applied Mathematics and Computation, vol. 180, no. 2, pp. 700-706, 2006.

[8] S. Zhang, "The existence of a positive solution for a nonlinear fractional differential equation," Journal of Mathematical Analysis and Applications, vol. 252, no. 2, pp. 804-812, 2000.

[9] N. Kosmatov, "A singular boundary value problem for nonlinear differential equations of fractional order," Journal of Applied Mathematics and Computing, vol. 29, no. 1-2, pp. 125-135, 2009.

[10] A. A. Kilbas, H. M. Srivastava, and J. J. Trujillo, Theory and Applications of Fractional Differential Equations, vol. 204, Elsevier Science B.V., Amsterdam, The Netherlands, 2006.

[11] M. A. Krasnoselskii, Positive Solutions of Operator Equations, P. Noordhoff, Groningen, The Netherlands, 1964.

[12] T. Qiu and Z. Bai, "Existence of positive solutions for singular fractional differential equations," Electronic Journal of Differential Equations, vol. 2008, article 146, 2008.

[13] X. Xu, D. Jiang, and C. Yuan, "Multiple positive solutions for the boundary value problem of a nonlinear fractional differential equation," Nonlinear Analysis. Theory, Methods \& Applications, vol. 71, no. 10, pp. 4676-4688, 2009.

[14] S. Zhang, "Nonnegative solution for singular nonlinear fractional differential equation with coefficient that changes sign," Positivity, vol. 12, no. 4, pp. 711-724, 2008.

[15] M. Feng, X. Zhang, and W. Ge, "New existence results for higher-order nonlinear fractional differential equation with integral boundary conditions," Boundary Value Problems, vol. 2011, Article ID 720702, 2011.

[16] D. Guo, Y. J. Cho, and J. Zhu, Partial Ordering Methods in Nonlinear Problems, Nova Science Publishers, New York, NY, USA, 2004.

[17] Z. Bai, "On positive solutions of a nonlocal fractional boundary value problem," Nonlinear Analysis: Theory, Methods \& Applications, vol. 72, no. 2, pp. 916-924, 2010.

[18] B. Ahmad and J. J. Nieto, "Existence of solutions for nonlocal boundary value problems of higher-order nonlinear fractional differential equations," Abstract and Applied Analysis, vol. 2009, Article ID 494720, 9 pages, 2009. 
[19] C. F. Li, X. N. Luo, and Y. Zhou, "Existence of positive solutions of the boundary value problem for nonlinear fractional differential equations," Computers \& Mathematics with Applications, vol. 59, no. 3, pp. 1363-1375, 2010.

[20] S. Zhang, "Positive solutions to singular boundary value problem for nonlinear fractional differential equation," Computers \& Mathematics with Applications, vol. 59, no. 3, pp. 1300-1309, 2010.

[21] Y. Zhao, S. Sun, Z. Han, and M. Zhang, "Positive solutions for boundary value problems of nonlinear fractional differential equations," Applied Mathematics and Computation, vol. 217, no. 16, pp. 6950-6958, 2011.

[22] J. Wang, H. Xiang, and Z. Liu, "Positive solution to nonzero boundary values problem for a coupled system of nonlinear fractional differential equations," International Journal of Differential Equations, vol. 2012, Article ID 186928, 12 pages, 2010.

[23] J. Zhao, P. Wang, and W. Ge, "Existence and nonexistence of positive solutions for a class of third order BVP with integral boundary conditions in Banach spaces," Communications in Nonlinear Science and Numerical Simulation, vol. 16, no. 1, pp. 402-413, 2011.

[24] W. Yang, "Positive solutions for a coupled system of nonlinear fractional differential equations with integral boundary conditions," Computers \& Mathematics with Applications, vol. 63, no. 1, pp. 288-297, 2012.

[25] X. Cheng and H. Lü, "Multiplicity of positive solutions for a (p1, p2) -Laplacian system and its applications," Nonlinear Analysis: Real World Applications, vol. 13, no. 5, pp. 2375-2390, 2012.

[26] X. Cheng and C. Zhong, "Existence of positive solutions for a second-order ordinary differential system," Journal of Mathematical Analysis and Applications, vol. 312, no. 1, pp. 14-23, 2005.

[27] X. Cheng and Z. Zhang, "Existence of positive solutions to systems of nonlinear integral or differential equations," Topological Methods in Nonlinear Analysis, vol. 34, no. 2, pp. 267-277, 2009.

[28] K. Deimling, Nonlinear Functional Analysis, Springer, Berlin, Germany, 1985.

[29] I. Podlubny, Fractional Differential Equations, Academic Press, New York, NY, USA, 1993. 


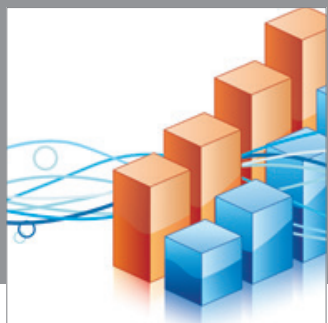

Advances in

Operations Research

mansans

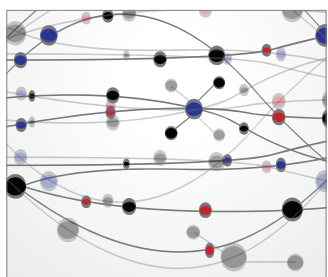

The Scientific World Journal
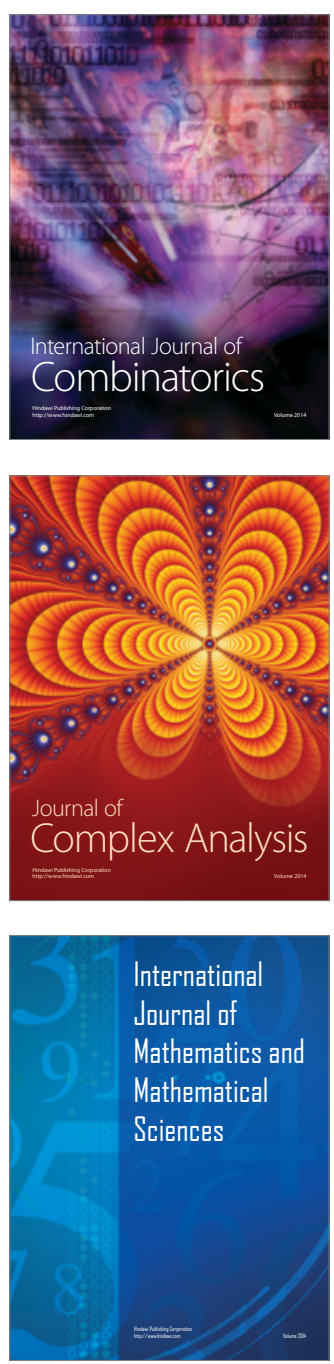
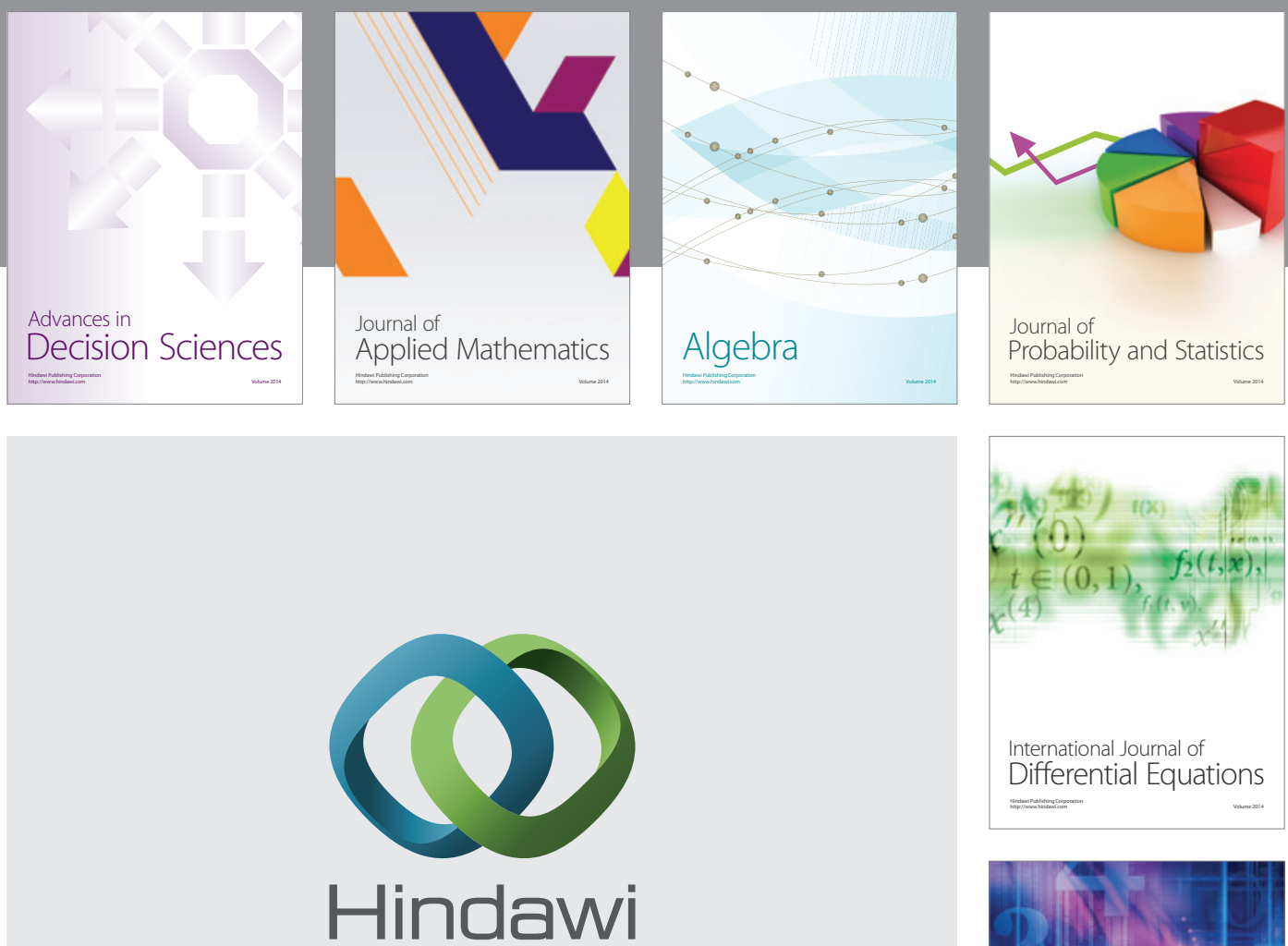

Submit your manuscripts at http://www.hindawi.com
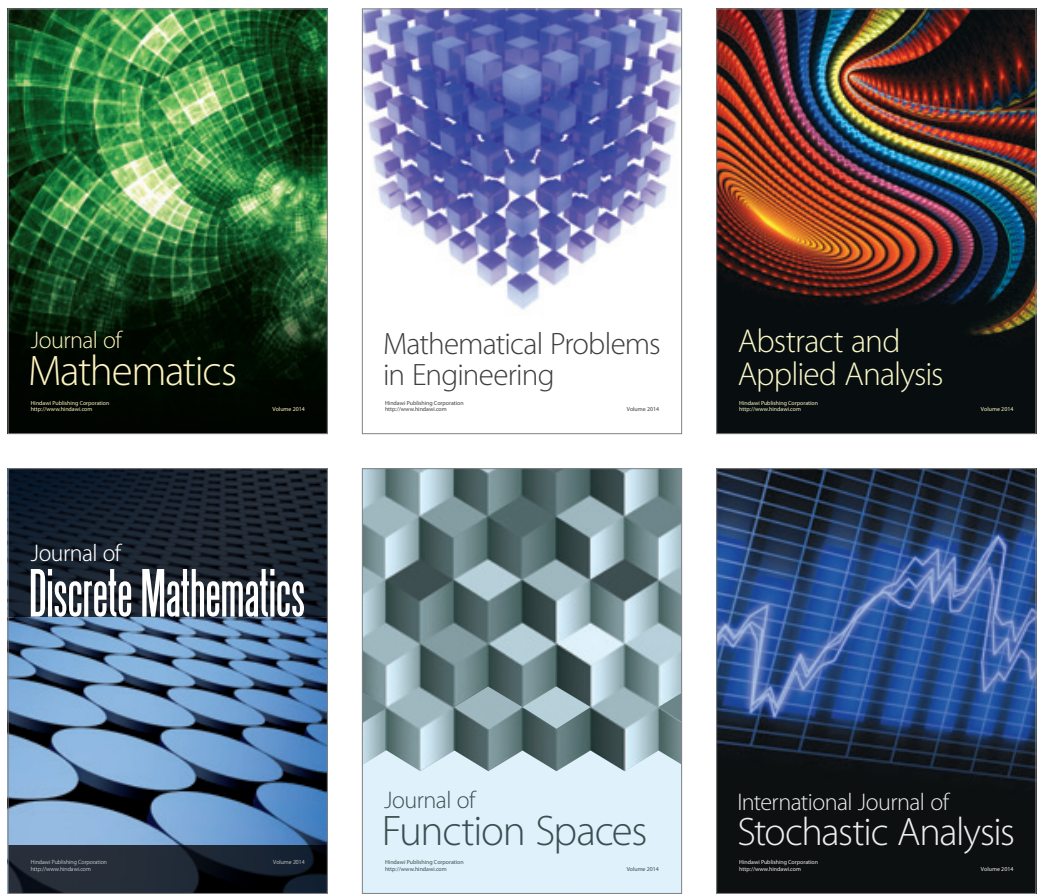

Journal of

Function Spaces

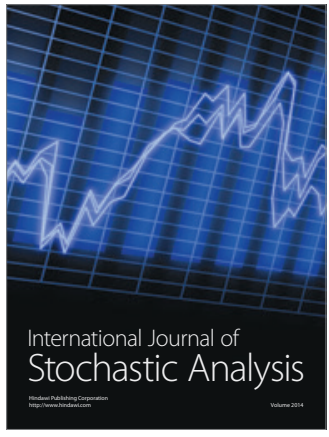

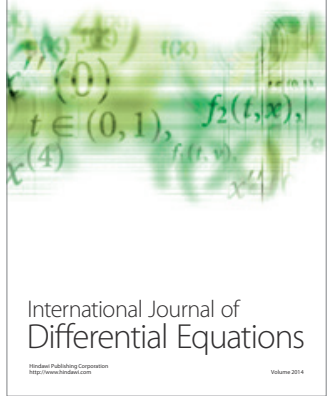
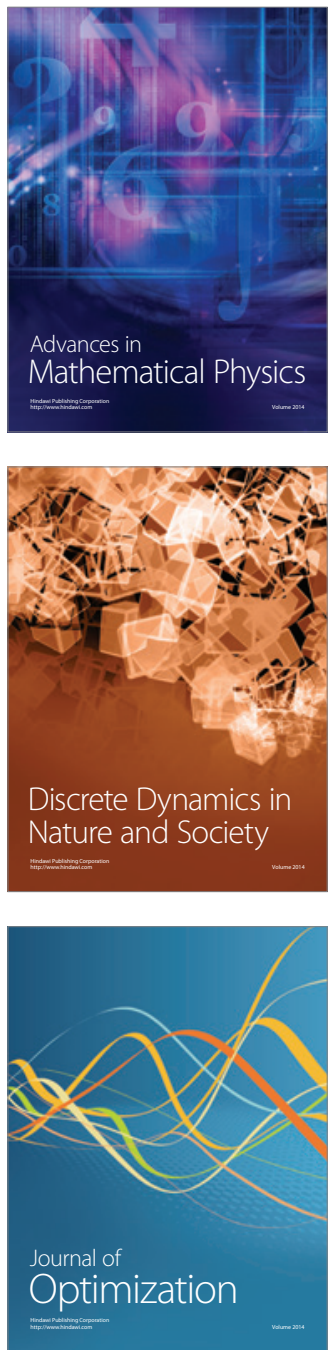